\title{
On a Correspondence Between Probabilistic And Fuzzy Choice Functions
}

\author{
Davide Martinetti • Susana Montes • \\ Susana Díaz • Bernard De Baets
}

\begin{abstract}
Probabilistic and choice functions are used to describe decision situations in which a certain degree of imprecision is involved. We propose a way to equate these two formalisms by means of residuum operators. Furthermore, a set of new rationality conditions for probabilistic choice functions is proposed and proved to be sufficient to ensure that the associated fuzzy choice function is rational.
\end{abstract}

Keywords Probabilistic choice - Fuzzy choice - Rationality conditions · Individual choice theory $\cdot$ Residuum operators

\section{Introduction}

Choice and preference are modelled in mathematics using respectively a choice function and a preference relation. If the (finite) universe of alternatives is denoted with $X$, then a choice function (in the classical sense) is simply a function $C$ that assigns to any non-empty set of available alternatives $S \in \mathcal{B}$ a non-empty subset $C(S) \subseteq S$ containing the alternatives that are chosen from $S$ (we denote with $\mathcal{B}$ the power set of $X$ without $\emptyset$ ). A preference relation $Q$ instead, is a function defined on the Cartesian product $X \times X$ that for any pair of alternatives $x$ and $y$ can take values 0 or 1 ; if $Q(x, y)=1$, then $x$ is considered at least as good as $y$, while if $Q(x, y)=0$ then $x$ is not preferred to $y$. One drawback of classical choice theory is that it does not account

Davide Martinetti

INRA Ecodeveloppment UR767, Avignon, France; E-mail: dmartinetti@paca.inra.fr

Susana Montes · Susana Díaz

Dept. Statistics and Operative Research, Oviedo University, Spain; E-mail: montes@uniovi.es - diazsusana@uniovi.es

Bernard De Baets

KERMIT, Dept. Mathematical Modelling, Statistics and Bioinformatics, Ghent University, Belgium; E-mail: bernard.debaets@ugent.be 
for those situations where choices or preferences are imprecise or ill-known. For example, experiments with repeated choices showed that an individual can choose differently when faced with the same set of available alternatives. In theory, it is a violation of rationality, but we know that such situation is quite common. Nevertheless, it cannot be modelled by classical preference relations, where, for any pair of alternatives, either one is strictly preferred to the other or they are indifferent (we intentionally exclude those alternatives that are incomparable). Hence the need of a more general framework in which similar situations are not considered to be pathological, but can at least be properly described. In this sense, we can mention at least two generalizations of classical choice theory: probabilistic (or stochastic) choice theory and the more recent fuzzy choice theory. The former is based on the intuition that the individual choice behaviour is probabilistic in nature; based on this assumption, a large literature has been developed, with contributions coming from different disciplines, such as economics and psychology [2-4,8-13, 15, 16, 23-25]. The second approach exploits the fact that imprecise situations can be conveniently described by means of fuzzy concepts, in which statements such as " $x$ is preferred to $y$ " or " $x$ is the chosen element from the set of alternatives $S$ " can be associated to a certain degree of truth. Fuzzy choice theory $[5-7,14$, $21,26,31]$, contrary to probabilistic choice theory, does not assumes that the behaviour of individuals is "fuzzy" in nature, but simply profits the modelling power of fuzzy set theory to accommodate for those situations in which choice and preference cannot be described in the classical way.

In this work we propose a way of constructing a fuzzy choice function from a given probabilistic choice function by making use of residuum operators. Our construction is inspired by a proposal of Fishburn [12] and it allows to construct several different fuzzy choice functions, according to the residuum operator of choice. This construction proves to be consistent (the generated fuzzy choice function is well-defined) and it is later used to propose new criteria for the rationality of probabilistic choice functions. The new rationality conditions are inspired by preceding proposals, such as the Luce Axiom of Choice ([16]) or the Weak Axiom of Stochastic Revealed Preference ([2]) and seem to mimic the contraction/expansion conditions $\alpha$ and $\beta$ and the Weak Axiom of Congruence, three milestones in classical choice theory (see for example $[1,27$, $29,30])$.

\section{Preliminaries}

The main concepts of probabilistic and fuzzy choice functions and preference relations are introduced in this section, together with a remainder on t-norms and residual residuum operators. 


\subsection{Probabilistic choice theory}

A probabilistic choice function (also called stochastic choice function) is a probabilistic version of a classical choice function. It first appeared in [16] as follows

Definition 1 Let $X$ be a finite set of alternatives and let $\mathcal{B}$ be the family of all subsets of $X$. A probabilistic choice function $P$ on $\mathcal{B}$ is a function that for any $T \in \mathcal{B}$ specifies exactly one finitely additive probability measure over the family of all subsets of $T$. Given a pair of sets $S$ and $T$, such that $S \subseteq T$, we denote with $P(S, T)$ the probability that the choice from the set of alternatives $T$ will lie in $S$.

The probabilistic choice function $P$ of a set $S \subseteq T$ is completely determined by its values $P(x, T)$, in the sense that $P(S, T)=\sum_{x \in S} P(x, T)$. In classical choice theory, from any choice function, a binary preference relation can be revealed (called revealed preference relation). The same occurs for probabilistic choice theory: in fact, to any probabilistic choice function $P$, we can associate a probabilistic preference relation, i.e. a binary relation $p: X \times X \rightarrow[0,1]$ such that $p(x, y)+p(y, x)=1$ for any pair of alternatives $x$ and $y$ in $X$ (in this work we will denote with upper-case $P$ the probabilistic choice function and with lower-case $p$ the associated probabilistic preference relation). The value taken by $p(x, y)$ indicates: the probability that the first alternative is preferred to the second one (if $p(x, y)>\frac{1}{2}$ ), the probability that the second is preferred to the first one (if $p(x, y)<\frac{1}{2}$ ) or that there is no preference between the two (if $p(x, y)=\frac{1}{2}$ ).

The most known and intuitively most compelling rationality condition for probabilistic choice function is usually called regularity condition RC (RG in [8], NC in [9]), which simply postulates that the probability of choosing $S$ from a set of alternatives $T(S \subseteq T)$ cannot increase if the set $T$ is expanded to a larger set $Z \supset T$.

Definition 2 ([8]) A probabilistic choice function $P$ on $X$ satisfies the Regularity Condition (RC) if, for any $S, T, Z$ in $\mathcal{B}$ such that $S \subseteq T \subset Z$, it holds that $P(S, T) \geq P(S, Z)$.

Regularity Condition can be interpreted as an adaptation to the stochastic framework of Condition $\alpha$ proposed by Sen in [30] (if $x \in S \subseteq T$ and $x \in C(T)$, then $x \in C(S))$. Another well-known rationality condition for probabilistic choice functions has been proposed by Luce in [16] and is usually referred as Luce's Axiom of Choice. Formally, it is composed of two parts:

Definition 3 ([16]) Let $P$ be a probabilistic choice function on $X$ and $p$ the associated probabilistic preference relation. It is said that $P$ satisfies Luce's Axiom of Choice if the following conditions hold: for any $S \in \mathcal{B}$ and $x \in S$ :

Part 1 If $p(x, y) \neq 0$, for any $y \in S$, then $P(x, X)=P(x, S) P(S, X)$.

Part 2 If $p(x, y)=0$, for some $y$ in $S$, then $P(S, X)=P(S \backslash x, X \backslash x)$. 
Part 2 is the least restrictive assumption: those alternatives $x \in S$ that are not chosen in some pairwise comparison with other alternatives in $S$ can be deleted from $S$ without affecting the choice probabilities. Part 1 states that the probability of selecting alternative $x$ from the universe $X$ is equal to the probability of selecting $x$ from a set $S$, multiplied by the probability of selecting $S$ from $X$. One consequence of Luce's Choice Axiom is that the probabilistic choice function $P$ satisfies the so-called constant ratio rule: for any $x, y$ in $S$, it holds that $\frac{P(x, S)}{P(y, S)}=\frac{P(x, X)}{P(y, X)}$.

Another condition for the rationality of the probabilistic choice function has been proposed by Bandyopadhyay et al. in [2]. It is inspired by the Weak Axiom of Revealed Preference (see Samuelson [28]) of classical choice theory (for any $S, T \in \mathcal{B}$ and $x, y \in S \cap T$, if $x \in C(S)$ and $y \in C(T)$, then $x \in C(T)$ ).

Definition 4 A probabilistic choice function $P$ on $X$ satisfies the Weak Axiom of Stochastic Revealed Preference (WASRP, for short) if, for any $S, T$ in $\mathcal{B}$ and any $A$ such that $A \subseteq S \cap T$, it holds that $P(A, T)-P(A, S) \leq P(S \backslash T, S)$.

The intuition behind this condition is the following: at the beginning $S$ is the set of available alternatives and $A \subseteq S$. The probability that the choice from $S$ will lie in $A$ is $P(A, S)$. Then the set of available alternatives changes from $S$ to $T$ and $A$ is also contained in $T$. If the new choice probability $P(A, T)$ is greater than $P(A, S)$, then it is reasonable to argue that this increase occurs only because the move eliminates some alternatives that were present in $S$ and are no longer available in $T$. But then the increase $P(A, T)-P(A, S)$ should not exceed the initial probability of choosing a subset in $S \backslash T$. Hence $P(A, T)-P(A, S)$ cannot be greater than $P(S \backslash T, S)$.

\subsection{Fuzzy choice theory}

Fuzzy choice theory has been proposed first by Banerjee [6] and further corrected and extended by Wang [32]. They develop a theory of choice where both preferences and choice functions are allowed to be fuzzy. Formally, a fuzzy choice function is a function $C: \mathcal{B} \rightarrow \mathcal{F}$, where $\mathcal{F}$ is a family of nonempty fuzzy subsets of $X$. For any $S \in \mathcal{B}, C(S)$ is a fuzzy set with non-empty support, where $C(S)(x)$ represents the extent to which alternative $x$ belongs to the set of chosen alternatives from $S$. The fuzzy set $C(S)$ should always be included into the available set $S$, i.e. $\operatorname{supp}(C(S)) \subseteq S$ and there should always exist an element $x$ for any $S \in \mathcal{B}$, such that $C(S)(x)>0$. A more general definition can be found in Georgescu [14], while a recent overview on fuzzy choice theory can be found in [33]. From a given fuzzy choice function $C$, a fuzzy preference relation $R_{C}$ can be revealed using

$$
R_{C}(x, y)=\bigvee_{\{S \mid x, y \in S\}} C(S)(x)
$$

A fuzzy preference relation $R$ is called $*$-regular, if it is reflexive (i.e. $R(x, x)=$ 1 , for any $x \in X)$, strongly complete (i.e. $R(x, y) \vee R(y, x)=1$, for any $x, y$ 
in $X$ ) and $*$-transitive (i.e. $R(x, z) \leq R(x, y) * R(y, z)$, for any $x, y$ and $z$ in $X$ ), where $*$ is a t-norm (see next Section 2.3). A crucial feature for a fuzzy choice function is the fact that the information contained in the fuzzy revealed preference relation $R_{C}$ and the fuzzy choice function $C$ itself are equivalent. This property is usually referred as normality:

Definition 5 A fuzzy choice function $C$ with fuzzy revealed preference relation $R_{C}$ is normal if $C(S)(x)=\bigwedge_{y \in S} R_{C}(x, y)$, for any $S \in \mathcal{B}$ and $x \in S$.

\subsection{Triangular norms and residuum operators}

When working in the fuzzy framework it is essential to define a set of operators that play the role of classical Boolean operators. In this paper, triangular norms and residuum operators are the operators of choice. We will use the notation $\wedge$ and $\vee$ for the minimum and maximum operators, respectively.

Definition 6 A triangular norm (t-norm for short) is a binary operation * on $[0,1]$ such that for any $a, b, c \in[0,1]$ the following properties are verified: i) commutativity: $a * b=b * a$; ii) associativity: $a *(b * c)=(a * b) * c$; iii $)$ monotonicity: if $a \leq b$, then $a * c \leq b * c$; iv) neutral element 1: $a * 1=a$.

The three most important t-norms are the minimum, $a *_{\mathbf{M}} b=a \wedge b$, the product, $a *_{\mathbf{P}} b=a \cdot b$ and the Eukasiewicz operator, $a *_{\mathbf{L}} b=(a+b-1) \vee 0$. A $\mathrm{t}$-norm is continuous if it is a continuous two-place function. A t-norm is called left-continuous if all partial mappings are left-continuous. To any t-norm $*$ we can associate another binary operator called residuum or implication, denoted $\rightarrow_{*}$, defined by: $a \rightarrow_{*} b=\sup \{c \in[0,1] \mid a * c \leq b\}$, for any $a, b \in[0,1]$. If the t-norm $*$ is left-continuous, then, for any $a, b \in[0,1]$, it holds that $a \leq b$ if and only if $a \rightarrow_{*} b=1$. Given a t-norm $*$ and its residuum operator $\rightarrow_{*}$, another operator called biresiduum can be defined: $a \leftrightarrow_{*} b=\left(a \rightarrow_{*} b\right) \wedge\left(b \rightarrow_{*} a\right)$. The biresiduum operator between $a$ and $b$ is often interpreted as a measure of how equal $a$ and $b$ are. It turns out that $a \leftrightarrow_{*} b=1$ if and only if $a=b$. In Table 2.3 we list some well-known t-norms and associated residuum and biresiduum operators.

\begin{tabular}{|c|c|c|}
\hline t-norm & Residuum & Biresiduum \\
\hline$a *_{M} b$ & $a \rightarrow_{M} b=\left\{\begin{array}{l}1 \text { if } a \leq b, \\
b \text { else }\end{array}\right.$ & $a \leftrightarrow_{M} b= \begin{cases}1 & \text { if } a=b \\
a \wedge b & \text { else }\end{cases}$ \\
\hline$a *_{P} b$ & $a \rightarrow_{P} b= \begin{cases}1 & \text { if } a \leq b, \\
b / a & \text { else }\end{cases}$ & $a \leftrightarrow_{P}=b \frac{a \wedge b}{a \vee b}$ \\
\hline$a *_{L} b$ & $a \rightarrow_{L} b= \begin{cases}1 & \text { if } a \leq b \\
1-a+b & \text { else }\end{cases}$ & $-|a-b|$ \\
\hline
\end{tabular}

Table 1 Three well-known t-norms and associated residuum and biresiduum operators

In this work we are supposing that the t-norm is chosen first and then residuum and biresiduum operators are derived from it according to the pre- 
vious definitions. In no case we will work with one t-norm $*_{1}$ and a residuum operator derived from another t-norm $*_{2}$. For this reason, in some cases we will avoid the usual notation of residuum and biresiduum operators $\left(\rightarrow_{*}\right.$ and $\leftrightarrow_{*}$ ) in which the dependence on the t-norm has to be made explicit.

\section{A correspondence between probabilistic and fuzzy choice functions}

Fishburn already addressed in [12] the lack of results on the connections between choice functions and probabilistic choice functions and hence he proposed a set of conditions that should be satisfied by the probabilistic choice function in order for the associated choice function to be rational. The same situation appeared in the framework of fuzzy choice theory and in the last years this gap has been filled with comprehensive studies on the connections between fuzzy and classical choice theory (see, amongst others, [6,14,17,21, $22,32,33]$ ). Surprisingly, there appears to be no literature on the comparison between fuzzy and probabilistic choice theory. We recently approached this issue in [18-20], where we proved some preliminary results. We followed an intuition proposed by Fishburn in [12], i.e. to construct a choice function from a given probabilistic choice function and then to find suitable conditions on the latter in order to ensure that the derived choice function is rational. One of the constructions proposed by Fishburn is the following:

Definition 7 ([12]) Given the probabilistic choice function $P$, a fuzzy choice function $C_{P}$ can be defined as

$$
C_{P}(S)(x)=\frac{P(x, S)}{\bigvee_{y \in S} P(y, S)} .
$$

Observe that the equation in Definition 7 can also be written using the residuum operator derived from the product t-norm (see Table 2.3):

$$
\begin{aligned}
C_{P}(S)(x) & =\frac{P(x, S)}{\bigvee_{y \in S} P(y, S)} \\
& =\bigwedge_{y \in S}(P(y, S) \rightarrow \mathbf{P} P(x, S)) .
\end{aligned}
$$

Inspired by this observation, we propose a novel construction of fuzzy choice functions from a given probabilistic choice function:

Definition 8 ([19]) Let $*$ be a left-continuous t-norm and let $P$ be a probabilistic choice function on $X$. A fuzzy choice function $C_{P}$ can be constructed using the following formula, for any $S \in \mathcal{B}$ and any $x \in S$,

$$
C_{P}(S)(x)=\bigwedge_{y \in S}(P(y, S) \rightarrow P(x, S)) .
$$


The fuzzy choice function $C_{P}$ derived from $P$ is always well-defined (independently from the chosen t-norm) and, for any $S \in \mathcal{B}$, there exists an element $x$ such that $C_{P}(S)(x)=1$ (in particular, $x$ is the alternative in $S$ for which $P(x, S)$ is the greatest).

\section{A new set of rationality conditions for probabilistic choice functions}

We turn now to the problem of finding suitable conditions on $P$ that can ensure that the associated fuzzy choice function $C_{P}$ satisfies some good properties. In particular, we will find inspiration by looking at the two conditions presented here in Definitions 3 and 4: Luce's Axiom of Choice and WASRP. Let us start with Luce's Axiom of Choice, namely, its first part expressed for any $S \in \mathcal{B}$ and any $x \in X$ as

$$
P(x, X)=P(x, S) P(S, X) .
$$

This implies immediately that, for any $S \in \mathcal{B}$ and $x \in X$,

$$
P(x, X) \geq P(x, S) P(S, X) .
$$

It is easy to prove that, for any $S \subseteq T \subseteq Z \in \mathcal{B}$, this last inequality implies

$$
P(S, Z) \geq P(S, T) P(T, Z) .
$$

We can rewrite this equation using the t-norm of the product $*_{\mathbf{p}}$ :

$$
P(S, Z) \geq P(S, T) *_{\mathbf{P}} P(T, Z),
$$

or, equivalently, using the property of residuum operators $a * b \leq c \Leftrightarrow a \leq b \rightarrow$ $c$, as

$$
P(T, Z) \rightarrow_{\mathbf{P}} P(S, Z) \geq P(S, T) .
$$

On the other hand, consider condition WASRP: for any $S \subseteq T \cap Z, S, T, Z$ in $\mathcal{B}$ it holds that:

$$
P(S, T)-P(S, Z) \leq P(Z \backslash T, Z) .
$$

In particular, if we choose $S$ such that $S \subseteq T \subseteq Z$, we can rewrite it as

$$
P(S, T)-P(S, Z) \leq P(Z \backslash T, Z)=1-P(T, Z) .
$$

Reordering this last equation as

$$
P(S, T) \leq 1+P(S, Z)-P(T, Z),
$$

we notice that it can be stated using the residuum operator derived from the Łukasiewicz t-norm:

$$
P(T, Z) \rightarrow_{\mathbf{L}} P(S, Z) \geq P(S, T) .
$$


Comparing the rewritings of Luce's Axiom of Choice and of WASRP, a clear pattern is visible: it seems that a rationality condition can be proposed using residuum operators in such a way that, for a given left-continuous t-norm * and associated residuum operator $\rightarrow$ and for any $S \subseteq T \subseteq Z$ in $\mathcal{B}$, it holds that

$$
P(S, T) \leq P(T, Z) \rightarrow P(S, Z) .
$$

Unfortunately, it is quite hard to interpret this equation, so we look for an equivalent statement with a more appealing formulation. Recall that Luce's Axiom of Choice is equivalent to the constant ratio rule. Hence we can rewrite Luce's Axiom using the biresiduum operator of the product t-norm:

$$
P(x, S) \leftrightarrow_{\mathbf{P}} P(y, S)=P(x, X) \leftrightarrow_{\mathbf{P}} P(y, X)
$$

and derive the following more general condition, holding for any left-continuous t-norm:

$$
P(x, S) \leftrightarrow P(y, S) \leq P(x, X) \leftrightarrow P(y, X) .
$$

In particular, since $\{x, y\}$ is the smallest set in $\mathcal{B}$ containing both $x$ and $y$, we can say that, for any $S \in \mathcal{B}$, it holds

$$
p(x, y) \leftrightarrow p(y, x) \leq P(x, S) \leftrightarrow P(y, S) .
$$

We can now state two new conditions for a probabilistic choice function based on this last reasoning.

Definition 9 Let $*$ be a left-continuous t-norm. A probabilistic choice function $P$ on $X$ satisfies the Weak Scalability Condition (WSC) if, for any $S, T$ in $\mathcal{B}$ such that $S \subseteq T$ and $x, y$ in $S$, it holds that

$$
P(x, S) \leftrightarrow P(y, S) \leq P(x, T) \leftrightarrow P(y, T) .
$$

A probabilistic choice function $P$ satisfies the Strong Scalability Condition (SSC) if, for any $S, T$ in $\mathcal{B}$ such that $S \subseteq T$ and $x, y$ in $S$, it holds that

$$
P(x, S) \leftrightarrow P(y, S)=P(x, T) \leftrightarrow P(y, T) .
$$

The interpretation of these conditions is quite simple: the degree to which two alternatives $x$ and $y$ are equally probable to be chosen is minimal when there are no more alternatives that can be chosen. If more elements are added to the set of possible choices, the probabilities of choosing $x$ or $y$ can only become more similar. Observe that if regularity condition $\mathrm{RC}$ of Definition 2 can be interpreted as a probabilistic version of Condition $\alpha$ of Sen [30], then the scalability conditions can play the role of Condition $\beta$ of Sen [30] in the probabilistic setting (i.e. if $x, y \in C(S)$ and $S \subseteq T$, then $x \in C(T)$ if and only if $y \in C(T))$.

We propose two last conditions inspired by the Weak Congruence Axiom ([27]) of classical choice theory (i.e. if $x \in S, y \in C(S)$ and $R_{C}(x, y)=1$, then $x \in C(S))$. 
Definition 10 A probabilistic choice function $P$ on $X$ satisfies the Weak Stochastic Congruence Axiom (WSCA) if for any $S \in \mathcal{B}$ and $x, y \in S$ such that $P(x, S)>P(y, S)$, it holds that $P(x, T) \geq P(y, T)$ for any other set $T$ containing $x$ and $y$.

It satisfies the Strong Stochastic Congruence Axiom (SSCA) if for any $S \in \mathcal{B}$ and $x, y \in S$ such that $P(x, S)>P(y, S)$, it holds that either $P(x, T)>$ $P(y, T)$ or $P(x, T)=P(y, T)=0$, for any other set $T$ containing $x$ and $y$.

The interpretation of these two conditions is the following: for condition WSCA, if there is at least one set $S$ where an alternative $x$ is strictly more probable to be chosen than another alternative $y$, then $x$ has to be considered at least as good as $y$ in all other sets that contain both alternatives. In other words, there cannot exist two sets $S$ and $T$ such that $x$ is strictly preferred to $y$ in $S$ and $y$ is strictly preferred to $x$ in the other set $T$. Condition SSCA is more demanding: it establishes that if there exists one set $S$ where an alternative $x$ is strictly more probable to be chosen than another alternative $y$, then $x$ is always strictly preferred to $y$, for any $T$ that contains both $x$ and $y$, unless both of them are unlikely to be chosen $(P(x, T)=P(y, T)=0)$.

\section{On the rationality of a fuzzy choice function constructed from a probabilistic choice function}

We know that in classical choice theory Conditions $\alpha$ and $\beta$ are equivalent to the joint normality of the choice function and regularity of the revealed preference relation, that in turn is equivalent to the Weak Congruence Axiom (WCA). In this section we study the consequences of assuming regularity and scalability conditions of the probabilistic choice function for the constructed fuzzy choice function, in an attempt to recover results similar to the classical case.

Let us prove first two auxiliary results.

Lemma 1 Let $*$ be a left-continuous $t$-norm. If the probabilistic choice function $P$ satisfies $S S C$ and WSCA, then for any $x, y$ in $X$ and $S \supseteq\{x, y\}$ it holds that

$$
p(x, y) \rightarrow p(y, x)=P(x, S) \rightarrow P(y, S) .
$$

Proof Consider an arbitrary pair of alternatives $x$ and $y$ and $S \in \mathcal{B}$ such that $\{x, y\} \subseteq S$. For $p(x, y)$ and $p(y, x)$, we only have three possibilities:

(i) If $p(x, y)<p(y, x)$, by WSCA, we have that $P(x, S) \leq P(y, S)$ and then trivially

$$
p(x, y) \rightarrow p(y, x)=1=P(x, S) \rightarrow P(y, S) .
$$

(ii) If $p(x, y)=p(y, x)$, then $p(x, y) \leftrightarrow p(y, x)=1$ and by SSC we have that

$$
1=p(x, y) \leftrightarrow p(y, x)=P(x, S) \leftrightarrow P(y, S) .
$$

Hence $P(x, S)=P(y, S)$ and Eq. (1) is trivially satisfied. 
(iii) If $p(x, y)>p(y, x)$, by WSCA, we know that $P(x, S) \geq P(y, S)$ and furthermore, using SSC, we have that

$$
\begin{aligned}
1 & >p(x, y) \rightarrow p(y, x) \\
& =p(x, y) \leftrightarrow p(y, x)=P(x, S) \leftrightarrow P(y, S) .
\end{aligned}
$$

Hence $P(x, S) \neq P(y, S)$, and in particular, by WSCA, $P(x, S)>P(y, S)$. Finally, using SSC, we have that

$$
\begin{aligned}
p(x, y) \rightarrow p(y, x) & =p(x, y) \leftrightarrow p(y, x) \\
& =P(x, S) \leftrightarrow P(y, S)=P(x, S) \rightarrow P(y, S) .
\end{aligned}
$$

In all three cases we proved that Eq. (1) is satisfied.

Lemma 2 Let $*$ be a left-continuous $t$-norm. If the probabilistic choice function $P$ satisfies WSC and SSCA, then for any $x, y$ in $X$ and $S \supseteq\{x, y\}$ it holds that

$$
p(x, y) \rightarrow p(y, x) \leq P(x, S) \rightarrow P(y, S) .
$$

Proof Consider an arbitrary pair of alternatives $x$ and $y$ and $S \in \mathcal{B}$ such that $\{x, y\} \subseteq S$. For $p(x, y)$ and $p(y, x)$ we only have three possibilities:

(i) If $p(x, y)<p(y, x)$, by SSCA, we have that either $P(x, S)<P(y, S)$ or $P(x, S)=P(y, S)=0$. In both cases $P(x, S) \rightarrow P(y, S)=1=p(x, y) \rightarrow$ $p(y, x)$.

(ii) If $p(x, y)=p(y, x)$, then by WSC we have that

$$
1=p(x, y) \leftrightarrow p(y, x) \leq P(x, S) \leftrightarrow P(y, S) .
$$

Hence $P(x, S)=P(y, S)$ and Eq. (2) is trivially satisfied.

(iii) If $p(x, y)>p(y, x)$, by SSCA, we have that either $P(x, S)>P(y, S)$ or $P(x, S)=P(y, S)=0$. In the second case $P(x, S) \rightarrow P(y, S)=1$ and Eq. (2) is trivially satisfied. In the first case, using WSC, we have that

$$
\begin{aligned}
p(x, y) \rightarrow p(y, x) & =p(x, y) \leftrightarrow p(y, x) \\
& \leq P(x, S) \leftrightarrow P(y, S)=P(x, S) \rightarrow P(y, S) .
\end{aligned}
$$

In all three cases we proved that Eq. (2) is satisfied.

The next result shows that the fuzzy preference relation $R_{C_{P}}$ revealed from $C_{P}$ can be written as a function of the probabilistic preference relation $p$, provided certain conditions are satisfied.

Proposition 1 Let $*$ be a left-continuous t-norm and $P$ a probabilistic choice function on $X$. If one of the following sets of hypotheses hold

Hypo. A $P$ satisfies conditions $S S C$ and $W S C A$;

Hypo. B $P$ satisfies conditions WSC, SSCA, RC and the t-norm * is the minimum, 
then the fuzzy preference relation $R_{C_{P}}$ revealed from $C_{P}$ can be written as:

$$
R_{C_{P}}(x, y)=p(y, x) \rightarrow p(x, y) .
$$

Proof Consider an arbitrary pair of alternatives $x$ and $y$ such that $x \neq y$ and recall the formula for computing the fuzzy revealed preference relation of a fuzzy choice function:

$$
R_{C_{P}}(x, y)=\bigvee_{\{S \in \mathcal{B} \mid x, y \in S\}} C_{P}(S)(x)
$$

Applying the formula for the construction of $C_{P}$ of Definition 8 to Eq. (4) we obtain:

$$
R_{C_{P}}(x, y)=\bigvee_{\{S \in \mathcal{B} \mid x, y \in S\}}\left(\bigwedge_{k \in S} P(k, S) \rightarrow P(x, S)\right) .
$$

The supremum in Eq. (5) is computed over the sets $S$ in $\mathcal{B}$ such that $x, y \in S$. This family of subsets of $\mathcal{B}$ can be split into $\{x, y\} \cup S_{x y}$, where $S_{x y}=\{S \in$ $\mathcal{B} \mid x, y \in S$ and $|S| \geq 3\}$ and then Eq. (5) becomes:

$$
R_{C_{P}}(x, y)=(p(y, x) \rightarrow p(x, y)) \vee \bigvee_{S \in S_{x y}}\left(\bigwedge_{k \in S}(P(k, S) \rightarrow P(x, S))\right)
$$

If $p(x, y) \geq p(y, x)$, then $p(y, x) \rightarrow p(x, y)=1$ and hence $R_{C_{P}}(x, y)=1=$ $p(y, x) \rightarrow p(x, y)$. Consider then the case $p(x, y)<p(y, x)$. Equation (6) implies that $R_{C_{P}}(x, y) \geq p(y, x) \rightarrow p(x, y)$. We next prove the opposite inequality, i.e. $R_{C_{P}}(x, y) \leq p(y, x) \rightarrow p(x, y)$, that, by Eq. (6), can also be proved by showing that

$$
\bigvee_{S \in S_{x y}}\left(\bigwedge_{k \in S}(P(k, S) \rightarrow P(x, S))\right) \leq p(y, x) \rightarrow p(x, y)
$$

If Нypo. A is satisfied, then using Lemma 1 we can write the right-hand part of Eq. (6) as:

$$
\begin{aligned}
\bigvee_{S \in S_{x y}}\left(\bigwedge_{k \in S}(P(k, S) \rightarrow P(x, S))\right) & \leq \bigvee_{S \in S_{x y}}(P(y, S) \rightarrow P(x, S)) \\
& =\bigvee_{S \in S_{x y}}(p(y, x) \rightarrow p(x, y)) \\
& =p(y, x) \rightarrow p(x, y) .
\end{aligned}
$$

Hence $R_{C_{P}}(x, y) \leq p(y, x) \rightarrow p(y, x)$.

If Hypo. B is satisfied and $p(y, x)>p(x, y)$, then the set $S_{x y}$ can be split into two subsets according to SSCA: 
(i) $S_{x y}^{>}=\left\{S \in S_{x y} \mid P(y, S)>P(x, S)\right\}$;

(ii) $S_{x y}^{=}=\left\{S \in S_{x y} \mid P(y, S)=P(x, S)=0\right\}$.

We will prove that in both cases $\bigwedge_{k \in S}(P(k, S) \rightarrow P(x, S)) \leq p(y, x) \rightarrow p(x, y)$, for any $S \in S_{x y}$. Assume $S \in S_{x y}^{>}$, then by Lemma 2 and the fact that the t-norm is the minimum, we have that

$$
p(x, y)=p(y, x) \rightarrow_{\mathbf{M}} p(x, y) \leq P(y, S) \rightarrow_{\mathbf{M}} P(x, S)=P(x, S) .
$$

Hence $p(x, y) \leq P(x, S)$, but by RC we know that $p(x, y) \geq P(x, S)$, so $p(x, y)=P(x, S)$ and the previous chain of inequalities implies $p(y, x) \rightarrow_{\mathbf{M}}$ $p(x, y)=P(y, S) \rightarrow_{\mathbf{M}} P(x, S)$.

If $S \in S_{x y}^{=}$, then there exists at least one alternative $z \in S$ such that $P(z, S)>$ 0 and hence $P(z, S) \rightarrow_{\mathbf{M}} P(x, S)=0$. Hence, in Eq. (6) the part $\bigwedge_{k \in S_{\bar{x}}=}(P(k, S) \rightarrow$ $P(x, S))$ becomes zero.

In both cases we proved that the right-hand part of Eq. (6) either is equal to zero or is equal to $p(y, x) \rightarrow p(x, y)$, hence $R_{C_{P}}(x, y)=p(y, x) \rightarrow p(x, y)$.

Proposition 1 shows that the fuzzy preference relation $R_{C_{P}}$ revealed from $C_{P}$ can be written as a function of the probabilistic preference relation $p$ derived from $P$. In this way, not only the choice functions are connected, but also their respective preference relations.

Remark 1 Combining Proposition 1 and Lemma 1 we immediately have that if $P$ satisfies SSC and WSCA, then $R_{C_{P}}(x, y)=P(y, S) \rightarrow P(x, S)$, for any $x, y \in S$ and any $S \in \mathcal{B}$ such that $\{x, y\} \subseteq S$.

We can now present the two main results of this work. They establish under which conditions the fuzzy choice function $C_{P}$ constructed from the probabilistic choice function $P$ is normal and the associated fuzzy revealed preference relation is $*$-transitive.

Proposition 2 Let $*$ be a left-continuous t-norm and let $P$ be a probabilistic choice function on $X$. If one of the following sets of hypotheses hold

Hypo. A $P$ satisfies conditions SSC and WSCA;

Hypo. B P satisfies conditions WSC, SSCA, RC and the t-norm * is the minimum,

then the associated fuzzy choice function $C_{P}$ is normal.

Proof To prove that $C_{P}$ is normal we need to show that $C_{P}(S)(x)=\bigwedge_{y \in S} R_{C_{P}}(x, y)$, for any $S \in \mathcal{B}$ and any $x \in S$. Consider an arbitrary $x \in X$ and $S \in \mathcal{B}$ such that $x \in S$.

If Hypo. A is satisfied, then, using Proposition 1 and Lemma 1, we can prove that

$$
\begin{aligned}
\bigwedge_{y \in S} R_{C_{P}}(x, y) & =\bigwedge_{y \in S}(p(y, x) \rightarrow p(x, y)) \\
& =\bigwedge_{y \in S}(P(y, S) \rightarrow P(x, S))=C_{P}(S)(x) .
\end{aligned}
$$


If Hypo. B is satisfied, we first prove that $\bigwedge_{y \in S} R_{C_{P}}(x, y) \geq C_{P}(S)(x)$. By definition of the fuzzy revealed preference relation $R_{C_{P}}$, we have that

$$
R_{C_{P}}(x, y)=\bigvee_{\{T \in \mathcal{B} \mid x, y \in T\}} C_{P}(T)(x) \geq C_{P}(S)(x)
$$

and hence that

$$
\bigwedge_{y \in S} R_{C_{P}}(x, y) \geq \bigwedge_{y \in S} C_{P}(S)(x)=C_{P}(S)(x) .
$$

Using Proposition 1 and Lemma 2, we can also prove the opposite inequality:

$$
\begin{aligned}
\bigwedge_{y \in S} R_{C_{P}}(x, y) & =\bigwedge_{y \in S} p(y, x) \rightarrow_{\mathbf{M}} p(x, y) \\
& \leq \bigwedge_{y \in S} P(y, S) \rightarrow_{\mathbf{M}} P(x, S)=C_{P}(S)(x)
\end{aligned}
$$

Combining the two parts, we then proved that $C_{P}(S)(x)=\bigwedge_{y \in S} R_{C_{P}}(x, y)$, i.e. $C_{P}$ is normal.

Proposition 3 Let $*$ be a left-continuous t-norm and $P$ a probabilistic choice function on $X$. If one of the following sets of hypotheses hold

Hypo. A $P$ satisfies conditions $S S C$ and $W S C A$;

Hypo. B $P$ satisfies conditions WSC, SSCA, RC and the t-norm * is the minimum,

then the fuzzy revealed preference relation $R_{C_{P}}$ is $*$-transitive.

Proof Let $x, y, z$ be three arbitrary alternatives in $X$. We want to prove that

$$
R_{C_{P}}(x, y) * R_{C_{P}}(y, z) \leq R_{C_{P}}(x, z) .
$$

If Hypo. A is satisfied, using Proposition 1 and Remark 1, we know that $R_{C_{P}}(x, y)=P(y, S) \rightarrow P(x, S), R_{C_{P}}(y, z)=P(z, S) \rightarrow P(y, S)$ and $R_{C_{P}}(x, z)=P(z, S) \rightarrow P(x, S)$, for any $S$ containing $x, y$ and $z$. Using the property of residuum operators $((a \rightarrow b) *(b \rightarrow c) \leq a \rightarrow c)$ we have that

$$
\begin{aligned}
R_{C_{P}}(x, z) & =P(z, S) \rightarrow P(x, S) \\
& \geq(P(z, S) \rightarrow P(y, S)) *(P(y, S) \rightarrow P(x, S)) \\
& =R_{C_{P}}(y, z) * R_{C_{P}}(x, y),
\end{aligned}
$$

that is exactly Eq. (8).

If Hypo. $\mathbf{B}$ is satisfied, by Proposition 1, we know that $R_{C_{P}}(x, z)=$ $p(z, x) \rightarrow p(x, z)$. Hence, if $p(z, x) \leq p(x, z)$, we immediately have that $R_{C_{P}}(x, z)=$ 1 and then Eq. (8) is trivially satisfied. Let us consider the case in which $p(z, x)>p(x, z)$ and $S=\{x, y, z\}$. By SSCA, there are two possibilities:

(i) $P(z, S)=P(x, S)=0$ or

(ii) $P(z, S)>P(x, S)$. 
In the first case we have that $P(y, S)=1$. By Lemma 2 and Proposition 1, we have that

$$
R_{C_{P}}(x, y)=p(y, x) \rightarrow_{\mathbf{M}} p(x, y) \leq P(y, S) \rightarrow_{\mathbf{M}} P(x, S)=1 \rightarrow_{\mathbf{M}} 0=0
$$

and then Eq. (8) is trivially satisfied.

In the second case, when $P(z, S)>P(x, S)$, suppose by absurdum that Eq. (8) is not satisfied, i.e.

$$
R_{C_{P}}(x, z)<R_{C_{P}}(x, y) *_{\mathbf{M}} R_{C_{P}}(y, z) .
$$

Using Proposition 1, Lemma 2 and the property of residuum operators $((a \rightarrow$ $b) *(b \rightarrow c) \leq a \rightarrow c)$ we have that

$$
\begin{aligned}
p(z, x) \rightarrow_{\mathbf{M}} p(x, z) & =R_{C_{P}}(x, z)<R_{C_{P}}(x, y) *_{\mathbf{M}} R_{C_{P}}(y, z) \\
& =\left(p(y, x) \rightarrow_{\mathbf{M}} p(x, y)\right) *_{\mathbf{M}}\left(p(z, y) \rightarrow_{\mathbf{M}} p(y, z)\right) \\
& \leq\left(P(y, S) \rightarrow_{\mathbf{M}} P(x, S)\right) *_{\mathbf{M}}\left(P(z, S) \rightarrow_{\mathbf{M}} P(y, S)\right) \\
& \leq P(z, S) \rightarrow_{\mathbf{M}} P(x, S) .
\end{aligned}
$$

Since the t-norm is the minimum, we have that

$$
p(x, z)=p(z, x) \rightarrow_{\mathbf{M}} p(x, z)<P(z, S) \rightarrow_{\mathbf{M}} P(x, S)=P(x, S),
$$

but this contradicts RC, i.e. $p(x, z) \geq P(x, S)$.

We can finally state the main result of this section.

Theorem 1 Let $*$ be a left-continuous t-norm and $P$ a probabilistic choice function on $X$. If one of the following sets of hypotheses hold

Hypo. A $P$ satisfies conditions $S S C$ and $W S C A$;

Hypo. B $P$ satisfies conditions WSC, SSCA, RC and the t-norm * is the minimum,

then the fuzzy choice function $C_{P}$ is normal and its fuzzy revealed preference relation $R_{C_{P}}$ is *-transitive.

\section{Conclusions}

Let us stress the importance of the results proved in this section. First of all, the possibility of constructing a fuzzy choice function from a given probabilistic choice function is fundamental, especially in a practical context. In fact, while fuzzy choice functions have been widely studied, it is still not clear how they can be observed in the real world. We agree with Banerjee [6] when he says that "[...] there may be problems of estimation, but fuzzy choice functions are, in theory, observable". With the proposed method we can rely on probabilistic choice functions, which are much easier to observe: they can be estimated by using the frequency of choice of an element in a set of alternatives, in a data set of repeated observations of choices on the same set of alternatives or be 
the results of more complicated multinomial discrete choice models, such as probit or logit models. Furthermore, if the observed probabilistic choice function satisfies certain conditions, then Theorem 1 ensures that the associated fuzzy choice function is normal and that its fuzzy revealed preference relation is $*$-transitive.

The estimation of probabilistic choice functions for big sets of alternatives can be problematic. In fact, even for a set $X$ with few alternatives, the number of subsets for which the probabilistic choice function needs to be known is $2^{|X|}-1$. Sets with more than 10 alternatives are already critical. Furthermore, the number of subsets $S$ of $X$ for which the probabilistic choice function $P$ can be directly observed is usually reduced. Thus, in the worst (and most probable) case, we can only observe $P$ on a reduced number of subsets of $X$. In this situation, the conditions of rationality involved in Theorem 1 can be used to reconstruct artificially the missing information on the subsets of $X$ that are not directly observed. In this way, we obtain a double benefit: we can complete the information on the non-observed sets and we are sure that the conditions needed for Theorem 1 are trivially satisfied.

Acknowledgements Davide Martinetti has received the support of the EU in the framework of the Marie-Curie FP7 COFUND People Programme, through the award of an AgreenSkills fellowship under grant agreement 267196. The research reported in this work has been partially supported by projects URBANSIMUL and EPIDEC.

\section{References}

1. K.J. Arrow. Rational choice functions and orderings. Economica 26, 121-127, 1959.

2. T. Bandyopadhyay, I. Dasgupta and P.K. Pattanaik. Stochastic revealed preference and the theory of demand. Journal of Economic Theory 84, 95-110, 1999.

3. T. Bandyopadhyay, I. Dasgupta and P.K. Pattanaik. Demand aggregation and the weak axiom of stochastic revealed preference. Journal of Economic Theory 107, 483-489, 2002.

4. T. Bandyopadhyay, I. Dasgupta and P.K. Pattanaik. A general revealed preference theorem for stochastic demand behaviour. Economic Theory 23, 589-599, 2004.

5. A. Banerjee. Fuzzy preferences and Arrow-type problems in social choice. Social Choice and Welfare 11, 121-130, 1994.

6. A. Banerjee. Fuzzy choice functions, revealed preference and rationality. Fuzzy Sets and Systems 70, 31-43, 1995.

7. C.R. Barrett and M. Salles. Social choice with fuzzy preferences - chapter twenty. In Handbook of Social Choice and Welfare, 367-389, Elsevier, 2004.

8. I. Dasgupta and P.K. Pattanaik. 'Regular' choice and the weak axiom of stochastic revealed preference. Economic Theory 31, 35-50, 2007.

9. I. Dasgupta. Contraction consistent stochastic choice correspondence. Social Choice and Welfare 37, 643-658, 2011.

10. P. Fishburn. Binary choice probabilities: on the varieties of stochastic transitivity. Journal of Mathematical Psychology 10, 327-352, 1973.

11. P. Fishburn. Models of individual preference and choice. Synthese 36, 287-314, 1977.

12. P. Fishburn. Choice probabilities and choice functions. Journal of Mathematical Psychology 18, 205-219, 1978.

13. P. Fishburn. Binary probabilities induced by rankings. SIAM Journal on Discrete Mathematics 3, 478-488, 1990.

14. I. Georgescu. Fuzzy Choice Functions: A Revealed Preference Approach, Springer, 2007.

15. R.D. Luce. A probabilistic theory of utility. Econometrica 26, 193-224, 1958. 
16. R.D. Luce. Individual Choice Behaviour. Wiley, New York, 1959.

17. D. Martinetti, I. Montes and S. Díaz. From preference relations to fuzzy choice functions, In Symbolic and Quantitative Approaches to Reasoning with Uncertainty, Lecture Notes in Computer Science, 594-605, 2011.

18. D. Martinetti, I. Montes, S. Díaz and S. Montes. A study on the transitivity of probabilistic and fuzzy relations. Fuzzy Sets and Systems 184, 156-170, 2011.

19. D. Martinetti, S. Díaz, S. Montes and B. De Baets. Bridging probabilistic and fuzzy approaches to choice under uncertainty. In proceedings of $8^{\text {th }}$ Conference of the European Society for Fuzzy Logic and Technology, EUSFLAT 2013.

20. D. Martinetti, S. Montes, S. Díaz and B. De Baets. Uncertain choices: a comparison of fuzzy and probabilistic approaches. In proceedings of $7^{\text {th }}$ International Summer School on Aggregation Operators, AGOP 2013.

21. D. Martinetti, B. De Baets, S. Díaz and S. Montes. On the role of acyclicity in the study of rationality of fuzzy choice functions. Fuzzy Sets and Systems 239, 35-50, 2014.

22. D. Martinetti, S. Montes, S. Díaz and B. De Baets. On Arrow-Sen style equivalences between rationality conditions for fuzzy choice functions. Fuzzy Optimization and Decision Making 13, 369-396, 2014

23. D.L. McFadden. Revealed stochastic preference: a synthesis. Economic Theory 26, 245$264,2005$.

24. S. Nandeibam. On probabilistic rationalizability. Social Choice and Welfare, 32, 425437, 2009.

25. S. Nandeibam. On randomized rationality. Social Choice and Welfare 37, 633-641, 2011.

26. G. Richardson. The structure of fuzzy preference: social choice implications. Social Choice and Welfare 15, 359-369, 1998.

27. M.K. Richter. Revealed preference theory. Econometrica 34, 635-645, 1966.

28. P.A. Samuelson. Note on the pure theory of consumer's behaviour. Economica 5, 61-71, 1938

29. A. Sen. Collective Choice and Social Welfare. Holden-day, 1970

30. A. Sen. Choice functions and revealed preference. Review of Economic Studies 38, 307317, 1971.

31. K. Sengupta. Choice rules with fuzzy preferences: some characterizations. Social Choice and Welfare 16, 259-272, 1999.

32. X. Wang. A note on congruence conditions of fuzzy choice functions. Fuzzy Sets and Systems 145, 355-358, 2004.

33. X. Wang, C. Wu and X. Wu. Choice functions in fuzzy environment: an overview. In 35 Years of Fuzzy Set Theory, Studies in Fuzziness and Soft Computing 261, 149-170, Springer, Heidelberg, 2010. 